\title{
The Complex Relations between Organization and Disorganization and Their Conceptual System
}

\author{
Kun Wu $\mathbb{D}^{1,2}$ Shan Zhang $\mathbb{D}^{2},{ }^{2}$ and Tianqi $W u(\mathbb{D})^{1,2}$ \\ ${ }^{1}$ International Center for Philosophy of Information of Xi'an Jiaotong University, Xi'an 710049, China \\ ${ }^{2}$ School of Humanities and Social Science of Xi'an Jiaotong University, Xi'an 710049, China \\ Correspondence should be addressed to Tianqi Wu; tianqi1262016@126.com
}

Received 26 October 2021; Accepted 15 November 2021; Published 28 November 2021

Academic Editor: Abdellatif Ben Makhlouf

Copyright $@ 2021$ Kun Wu et al. This is an open access article distributed under the Creative Commons Attribution License, which permits unrestricted use, distribution, and reproduction in any medium, provided the original work is properly cited.

\begin{abstract}
For improving and developing self-organization theory in contemporary complex information systems theory, it is necessary to reinterpret related concepts and their relations and construct a conceptual system related to self-organization theory. Based on the latest research results, this article specifically discusses concepts of organization and disorganization, static organization and dynamic organization, static disorganization and dynamic disorganization, organizing and disorganizing, self-organization and other-organization, self-disorganization and other-disorganization, and their relations. Further, it explores the mechanism of organization and disorganization, the compatibility of organization and disorganization, feedback and mutual other-disorganization in interaction, constraint and freedom of organization and disorganization, complexity of restraint and external factors, and relations between "the good" and "the evil" in restraint and freedom. On this basis, a diagram of the conceptual system of organization and disorganization, including four levels and their relations, is presented.
\end{abstract}

\section{Introduction}

Self-organization theory is an important research field in the development of contemporary complex information systems theory. Associated with the construction of this theory, academia has advanced a series of basic concepts, relations, and theorems. These include concepts of organization and disorganization, self-organization and other-organization, which are the most fundamental, and related concepts including order and disorder, ordering and disordering, and evolution and degradation. To better study, develop, and improve self-organization theory, it is necessary to clearly define and explain many related concepts and their levels, relations, characteristics, and principles.

A pioneer of self-organization theory, the founder of dissipative structure theory, Belgian scientist Ilya Prigogine (1917-2003), first proposed and used the concept of selforganization to describe the process and mechanism of spontaneously producing ordered structures [1].

Another founder of contemporary self-organization theory, German physicist and founder of synergetics,
Hermann Haken (1927-) distinguished the two concepts of organization and self-organization. He noted, "consider, for example, a group of workers. We then speak of organization or, more exactly, of organized behavior if each worker acts in a well-defined way on given external orders, i.e., by the boss." "We would call the same process as being self-organized if there are no external orders given but the workers work together by some kind of mutual understanding, each one doing his job so as to produce a product" [2].

Mr. Dongsheng Miao raised objections to the classification standard of Haken's organization and self-organization because it confuses the levels of the concepts of organization and self-organization. Organization should be the superior concept of self-organization, and the opposite concept of self-organization should be other-organization. Mr. Miao believes that the organization mentioned by Haken should actually be other-organization [3].

Professor Tong Wu and others advanced the concepts of disorganization and disorganizing, organizing, and beingorganized $[4,5]$. 
Although the concept of being-organized proposed by Professor Tong $\mathrm{Wu}$ and others resembles the concept of other-organization proposed by Mr. Dongsheng Miao, this article uniformly adopts the concept of other-organization when referring to related terms considering that it is more appropriate to use the two corresponding concepts of "other" and "self" to describe a contradictory phenomenon and process.

Based on relevant scholars' opinions, this article attempts to construct a conceptual system related to the concepts of organization and disorganization and concisely summarizes the meanings and complex relations of related concepts.

\section{Organization and Disorganization and Their Static and Dynamic Types}

2.1. Organization and Disorganization. Organization and disorganization are a pair of categories that describe the state of system structure. The structure pattern with order is the basic meaning of the concept of organization. Correspondingly, the meaning of disorganization should be the structure pattern with disorder.

If described in the context of information science, the greater system's entropy, the higher the degree of disorganization, and the smaller the entropy, the more organized the system. In this way, organization and disorganization actually describe the system's order and disorder, which is a pair concept. However, it should be noted that a simple distinction between organization and disorganization differences based on entropy may not work in extreme cases. This involves the criterion of static organization and static disorganization.

2.2. Static Organization and Dynamic Organization. The ordered structure of the system can be maintained in two different ways: one is the relative stability of the position of the system elements, such as the ordered structure of the "lattice" of gemstones, the ordered framework and hierarchical structure of a building, and the other is that although the elements of the system are constantly replacing and changing, its overall orderly mode is relatively stable, such as the vortex in a river and the maintenance of life in exhaling the old and inhaling the new.

Two different organizations are thus distinguished: static and dynamic organizations. The former is "dead structure," and the latter is "dynamic order." Generally, the ordered structure of a static organization can be maintained in a relatively isolated or closed state. In contrast, the ordered structure of a dynamic organization can only be maintained under an open background of moderate interaction with the environment.

2.3. Static Disorganization and Dynamic Disorganization. Not only are there static or dynamic organizations, there are also two types of disorganization, static or dynamic.

The maintenance of a static disorganization system is also based on the relative stability of the system's microelements, which differs from the static organization in that there is no substantial interaction between the microelements of the system, or there is only some weak linear interaction. For example, the third law of thermodynamics describes the situation when the thermodynamic system is in a state of absolute zero (0k). In this state, all microscopic particles are in an absolutely motionless state, and it is impossible for particles to interact. At this time, it is invalid to judge by the amount of entropy because system entropy is zero. While such a state is only ideal, it may never be achieved. Another example of static disorganization is the disorganization of sand. In this case, although there is a weak linear interaction between each sand grain and its neighbor, long-range coherence across the elements cannot be formed. Similarly, there are amorphous solids that are irregularly shaped, such as glass, which only has short-range order and does not have long-range order.

The maintenance of a dynamic disorganization system is realized through the free activities of microelements, but these elements' free activities cannot form an overall ordered macro difference pattern (e.g., the equilibrium system explained via thermodynamics). In such a system, although all system microscopic particles are in free Brownian motion, it is impossible to form a macroscopic ordered structure as a whole. In addition, the difference between a dynamic disorganization system and a dynamic organization system is that the latter must be produced and maintained in an open background, while the former may be formed and maintained in an isolated state.

\section{The Formation of Organization and Disorganization}

3.1. Organizing and Disorganizing. The concepts of organization and disorganization describe the orderliness and disorderliness of a system, respectively. However, a system's orderly or disorderly pattern can be generated, changed, and transformed into each other under specific conditions. The pair of categories that describe this process of generation, change, and mutual transformation are organizing and disorganizing. Obviously, as far as the process is concerned, the two categories of organizing and disorganizing belong to the process of dynamic organization or dynamic disorganization.

Organizing is the evolution process of a system from a disorganized state to an organized state, or from a low-level organizational state to a high-level organizational state. On the contrary, disorganizing is the evolution process of the system from a high-level organizational state to a low-level organizational state, or from an organizational state to a disorganized state. The former corresponds to the process of system organization enhancement, that is, the evolution process of system structure from disorder to order, from low-level order to high-level order; the latter corresponds to the process of organizational degradation, that is, the evolution process of the system from high-order degradation to low-order, or from orderly degradation to disorder. Obviously, these two processes also correspond to two branches of evolution: anagenesis and degeneration. 
3.2. Self-Organization and Other-Organization. What specific mechanism and method are used to realize the process of generating and maintaining an ordered or disordered system described by organization and organizing? This involves the problem of self-organization and otherorganization.

In general, the generation of all ordered structures is a dynamic organization and organizational process. Since all organization and organizing processes can only be generated under an open background, the description of this generation mechanism can be interpreted from the content, mechanism, mode, and process of interaction between external and internal factors. Self-organization and other-organization are categories describing the conditions, contents, mechanisms, modes, and processes of internal and external factors. In this way, both self-organization and other-organization are only aimed at ordered structural systems with the generation, maintenance, and transformation mechanisms.

According to the usual interpretation, we can define other-organization as the process of directly introducing model information from outside and constructing the system model accordingly. Essential to understanding the concept of other-organization is that modes are input from outside rather than generated spontaneously within the system. For example, workers work according to external instructions issued by the foreman, leading to joint production behavior; a crystal grows according to the implanted crystal embryo pattern; a factory carries out replication production in accordance with the imported product model; and reform of a unit or institution based on the successful experience of existing units or institutions also belongs to the scope of other-organizations [6].

Mr. Dongsheng Miao once attempted to combine the other-organization with the methods of cybernetics for interpretation. In his view, "In the process of system organization, the organizer and the being-organized need to be clearly distinguished. The organizer has organizational power and issues control instructions. The being-organized accepts and executes such instructions, so as to form and maintain an orderly structure of the system, or change the orderly structure of the system, and then change system's attributes, functions, behavior patterns, etc., that is otherorganization" [7]. Mr. Miao's mode of interpretation is reasonable and precise. From this, we can conclude that the cybernetics theory founded by the US mathematician Norbert Wiener (1894-1964) is actually a theory about other-organization [8]. In human society, all the top-down command, management, and control processes are the processes of other-organization.

Similarly, self-organization can be defined as the process whereby a system spontaneously forms an internal ordered structure in an open context. The key to understanding the concept of self-organization is that the pattern is spontaneously generated internally, rather than directly given from outside the system. In fact, the generation of any new ordered structure pattern is achieved through self-organization, which is the general mechanism for creation of new models. Examples of this include the production process of collective operation by workers through active cooperation and tacit action, the process of generating laser wave train spontaneously, the process by which a living body constructs its own flesh, blood, body, and organs by absorbing external substances and energy.

Haken, the founder of synergetics, once defined: "We shall say that a system is self-organizing if it acquires a spatial, temporal or functional structure without specific interference from the outside. By "specific" we mean that the structure or functioning is not impressed on the system, but that the system is acted upon from the outside in an nonspecific fashion" [9].

Both other-organization and self-organization are processes through which systems obtain their spatial, temporal, or functional ordered structures. Moreover, both depend on the openness of the system. The difference between the two is only in the nature of the external input factors. In the actual process of system evolution, there is no absolute boundary between self-organization and other-organization. Therefore, for convenience, some scholars discuss self-organization and other-organization systems together, so the term "self-other organization" appears [10].

According to self-organization theory, two processes can be described in a unified viewpoint. In fact, the process of other-organization can be regarded as the quantitative replication or diffusion of new patterns created in the selforganization process. Thus, creating a new ordered structure of all matter or information is a self-organizing process of qualitative change or transition of things. The process of other-organization can be regarded as the quantitative diffusion or further amplification of the self-organization process.

It is worth emphasizing that the process of creating an orderly model of a system, whether through other-organization or self-organization, must be realized under open conditions. However, this does not mean that an open background must guarantee the maintenance of all orderly patterns. In fact, after some structures are formed in, they may be kept in relatively isolated or closed conditions for a period of time. Examples include a natural "lattice" developed according to the input pattern, a building constructed according to a given pattern, and a rock or stratum structure formed spontaneously under the disturbance of relevant environmental factors. This kind of phenomenon can be regarded as the transformation process from dynamic organization to static organization. However, in most cases, the maintenance of the orderly pattern formed through dynamic organization also needs to be maintained dynamically under the open background, for example, the vortex in the river, the ordered structure of the living organism, the continuous emission of lasers, the maintenance of the pattern formed by the Bénard flow, and so on.

3.3. Self-Disorganization and Other-Disorganization. In the literature of general self-organization theory, only the concepts of self-organization and other-organization are involved, and no one has proposed the concepts of selfdisorganization and other-disorganization. 
In fact, the generation and maintenance of the disorganization state also need corresponding conditions, mechanisms, and processes. The concepts that describe this condition, mechanism, and process are self-disorganization and other-disorganization. Similar to the description of selforganization and other-organization, self-disorganization and other-disorganization can also be explained from the content, mechanism, method, and process of interaction between external and internal factors.

Other-disorganization can be defined as the process of the formation and maintenance of the disordered state of the system caused by the direct intervention of specific external factors, for example, the state of war in the country caused by foreign invasion; the extinction of one or some animals and plant populations due to the erosion of alien species; the deterioration of the Earth's natural ecological environment caused by human blind exploitation, and so on.

Self-disorganization can be defined as the process of forming an internal disordered structure of the system spontaneously. For example, national civil strife is caused by the intensification of internal contradictions, the process of aging, and death experienced by organisms spontaneously; the spontaneous degradation process of the system's ordered structure, and so on. Moreover, the process of "self-disorganization" is the most common state in an entropy-increasing universe, such as the accelerating separation of galaxies, the burning and death of stars, the heat death of the universe, and so on. On the contrary, self-organization can only occur in a few small areas.

The standard of distinguishing self-disorganization and other-disorganization is similar to self-organization and other-organization. They are all classified according to direct input from the outside or spontaneous formation from the inside. However, there is a difference between the relevant conditions of self-disorganization and self-organization. All self-organization processes that generate orderly structures must be open to the outside world, while the system for selfdisorganization processes that generate disordered structures can be open or isolated and closed. For example, the equilibrium system explained in thermodynamics is a disordered state in which the entropy is close to the maximum spontaneously formed in an isolated system.

\section{The Compatibility of Organization and Disorganization and Their Generation}

4.1. The Compatibility between Organization and Disorganization. In the real world, the internal structure of all specific systems cannot wholly reach $100 \%$ organization or $100 \%$ disorganization; that is, it is impossible to achieve complete order or complete disorder. The internal structure of any real system is a complementary and intertwined compatible state of organization and disorganization, which means the internal structure is partially organized or partially disorganized. The difference lies only in the quantitative degree of organization or disorganization.

The third law of thermodynamics assumes an absolutely disorganized or absolutely disordered system state: absolute zero. However, this law also emphasizes that absolute zero is impossible to achieve. Modern cosmology does not support the existence of absolute zero in the universe, posing a problem. The crystal structure of the static ordered system mentioned above assumes that when it is infinitely close to absolute zero, it should be in an almost absolute static ordered state, and the crystal particles should be almost motionless. Consequently, the ordered crystal structure should be maintained almost indefinitely. However, from the third law of thermodynamics, this state is almost absolute disorder, a paradox. Therefore, we should measure the order and disorder and entropy increase and entropy decrease of things from another angle. These measurements should not be bound to matter's spatial structure and components but related to the long-range correlation and long-range information flow among the system elements. From this point of view, the long-range correlation of a static ordered system is not strong, and there is no complex interaction and long-range information flow. Whether this organizational system should be classified as an ordered system with high entropy remains to be discussed.

The example of a disorganized system is often referred to as "a plate of scattered sand." Even in such a system, there is also force interaction between adjacent sand particles, and such interaction also has the characteristics of the shortrange organization.

The equilibrium state with the maximum entropy referred to in the second law of thermodynamics is usually regarded as the most disordered or disorganized state. However, there is a local deviation from the equilibrium state created by accidental fluctuation caused by particle disturbance on the microlevel even in such equilibrium state. Therefore, the so-called entropy maximum state does not exist.

As for the idealized and completely orderly system structure, it is impossible in the real world because the internal structure of any system is multiple, hierarchical, and complex. Complete order or $100 \%$ organization means that many elements at all system levels can only be related according to a rigid and unique relationship, which is impossible. The elements in the system obey the nature of the overall relationship and have some freedom in their own behavior. As a result, there are various levels of relations within the system.

Due to the differences, diversities, and hierarchies among the system elements, it is impossible to unify them all in a single rigid and unchanging relation. However, if system elements are unified, the differences, diversities, and hierarchies would all disappear. In this regard, any thing organized in absolute order is a thing without an internal difference. Such a thing is the "atom" without internal structure, impenetrable, and no longer changing as envisaged by ancient Greek atomism. Such an entity can only be an absolutely isolated thing. The lack of internal structure means that it is impossible to produce any intermediary outwards due to the adjustment of the internal structure. Because it is impenetrable and no longer changes, it is impossible to interact with the external environment. Since a system must have an internal structure, such absolute isolation cannot be a system. Everything in the world exists 
through interaction. The minimum entropy, the maximum entropy, and the completely ordered system state all belong to the category of nonbeing that can only be directed by human imagination subjective information [11]. Therefore, such absolute isolation without internal structure cannot exist in the real world. From another perspective, if it did exist, we would be unable to understand it because it could not interact with anything else.

4.2. The Compatibility between Self-Organization and Other-Organization and Self-Disorganization and OtherDisorganization. We have discussed the different generation approaches and methods of self-organization and otherorganization, self-disorganization, and other-disorganized, while explaining the specific system organizing and disorganizing mechanism. The description of different approaches and methods is relative to the different roles and properties of system external and internal factors. Specifically, if the system generation mode is directly input from the outside, it is other-organization or other-disorganization; if the system generation mode is spontaneously generated internally, it is self-organization or selfdisorganization.

Except for the disorganized system formed under isolated conditions (e.g., thermodynamic equilibrium states), all self-organization and other-organization and self-disorganization and other-disorganization are carried out under open conditions. In the dynamic evolution of things, system internal and external factors are always in synergy. Therefore, both external and internal factors contribute to the generation of the internal structure of the system. In some cases, a clear distinction can be made between external effects and internally generated patterns, but this distinction is not very strict in more cases.

On the surface, any other-organized or other-disorganized internal structure mode is directly input from the outside. However, the transformation of the external input structure mode into the internal structure also requires the coordination of internal factors. If the same external mode acts on different systems, the specific modes generated within the systems will be different due to the systems' different internal properties and characteristics. In such cases, we would rather conclude that the generation of the system's internal structure is not pure selforganization (self-disorganization) or pure other-organization (other-disorganization) but a combination or compatibility of self-organization (self-disorganization) and other-organization (other-disorganization).

For example, the process of learning and receiving education is a compatible way of integrating other-organization and self-organization. Learning book knowledge and listening to teachers are all other-organizational behaviors that input information from the outside. However, any external input knowledge can only form its own knowledge structure through the receiver's internal selection, understanding, and processing. Due to differences in people's internal knowledge structure, cognition, and abilities, even if they accept the same input of external knowledge, they will have very different or even opposite learning effects. As a result, the external input knowledge information is reconstructed through internal self-organization. Therefore, the learning process can only be a compatible process with other-organization and self-organization.

\subsection{Feedback and Mutual Other-Organization in Interaction.} Human practice can also be regarded as a process of integration, compatibility, complementation, and transformation of other-organization and self-organization. Human practice starts with the idea of purpose and plan, and any purpose and plan is a mode of regenerating information constructed by self-organization. When people transform their purpose into the structural information of the object through the practice of a specific plan, this is an other-organizational process for the realization of purposeful information. In addition, a feedback loop interaction is established among the practice subject, the practice process, and the practice object in the entire planning practice. Through the feedback loop of the interactive information flow, a relation of mutual other-organization is established. In this regard, any control process with a feedback loop will be an organizational process with a mutual other-organization nature.

Further analysis finds that the generation of any selforganization mode is a process of establishing a nonlinear feedback loop interaction between the system's elements and between the elements and the whole. In this way, the generation of the whole self-organization mode of the system depends on internal elements and mutual otherorganizational relations between the elements and the whole. This whole self-organization is realized through individual mutual other-organization, which fully reflects the complementary, compatible, and unified nature of self-organization and other-organization.

\section{Organization and Disorganization: Constraints and Freedoms}

5.1. Constraints and Freedoms and Their Limits. As mentioned earlier, organization and disorganization describe the orderliness and disorderliness of system structure. In fact, this order or disorder, organization or disorganization is realized through the relation between constraints and freedoms of system elements.

Generally, any organization process that increases the degree of order increases the constraints on elements; that is, it limits the freedom of elements' behavior. Any disorganized process that increases the degree of disorder reduces the constraints on the elements; that is, it increases the freedom of elements' behavior.

There are two limits. One is an extremely orderly organizational state where all system elements are completely and absolutely operated in accordance with the overall rules. All elements (individuals) have lost any independent behavior choice ability. The other limit is the disorganized state of extreme disorder, in which all elements are free and independent in their own way, completely and absolutely free from the constraints of the overall relation of the system. 
For example, the crystal structure mentioned above is infinitely close to absolute zero, and each element state in the system is infinitely close to static. This almost absolutely ordered organizational state cannot be called a system. The extremely disordered disorganized state itself is a typical nonsystem state.

Either of these two extremes is a dispelling of the system itself, resulting in a system that can no longer exist as a system. An absolutely rigid ordered system completely eliminates internal differences, while a system with completely independent and free elements loses relations between the elements. Therefore, such systems will be transformed into unstructured nonsystems, because the structure of systems requires internal differences between elements. In reality, such things cannot exist, because any thing that has lost the internal difference relations, or the elements are completely in an independent and free state, will be a dead unchanging thing. Given that it is absolutely independent or has no internal differences and no longer changes, such a thing cannot interact with any thing and thus cannot manifest itself through interaction. A thing that cannot manifest itself can never be recognized. If so, such a thing can only be a pure "nothing" that cannot exist, or a pure "nonbeing" from the perspective of epistemology [12].

The French scholar Edgar Morin emphasized the following: "All unions inevitably lead to constraints" and "Constraints are to limit and bind parts. These restrictions, limitations, and constraints either deprive or suppress the advantages or attributes of each part" [13]. "All organizations that determine and develop hierarchy and labor division will determine and develop constraint, oppression, and slavery" [13]. This is why Wiener opposes the application of the principle of strong control to the fields of human thinking, social order, and scientific development, and calls the "orderly country with pre-designated individual social functions" as "ant society" [14].

\subsection{Similarities and Differences between Self-Organization} Constraints and Other-Organization Constraints. In discussing the differences between self-organizations and other-organizations in forming constraints on system individuals, Professor Tong Wu believes that "The sub-system in self-organization is a kind of regular, parametric, intangible, and influential domination (similar to the invisible hand in the economic process), while the sub-system in the being-organized system is the total system rigid, irregular, and tangible domination and servitude". Professor Tong Wu emphasizes that the elements of a self-organized system are constrained by the whole, with some spontaneity and self-consciousness, while the elements of a system other-organized are constrained by the whole, with some compulsion and passivity. However, by whatever means, the resulting constraint of the whole to the part is a type of restriction, oppression, deprivation, and enslavement. After some spontaneous restraint becomes a habit, the situation in which individuals are restricted, oppressed, deprived, and enslaved may be more ambiguous, deceptive, and paralytic than the restriction, oppression, deprivation, and enslavement forced through other-organization.
5.3. Complexity of Constraints and External Factors. The similarities and differences between self-organization and other-organization constraints also involve an ancient philosophical problem: the relation between external and internal causes. A traditional view holds that in the development of things, the internal factors are the basis and decisive, and the external factors are the condition that works through the internal cause. In fact, this view simplifies the complex relationship between internal and external causes and has a mechanistic characteristic of unipolar thinking.

In the process of self-organizing constraints, the internal basis plays a leading role in the construction of new models. In the process of other-organizing constraints, external factors directly become the leading factor in the construction of new models. In the former scenario, internal factors play the role of the agents and initiators in the formation of new structures. In the latter scenario, the relation is reversed. External factors are agents and initiators, while internal factors are dominated and driven.

In general, the effect of external factors on the system can be placed in three cases. First, the nonspecific constraints imposed on the system from the outside can provide suitable conditions for the orderly development of the system itself. The system will construct the overall orderly mode suitable for external conditions through self-organization. Second, the nonspecific constraints imposed on the system from outside cannot provide corresponding support conditions for maintaining the system's orderly mode. At this time, the system's existing orderly structure will lose its stability, and the system will face the possibility of a new evolution direction. According to the nature and strength of external constraints, it will either develop towards a more orderly mode or cause the degradation of the existing orderly mode and move towards a disorderly evolution direction. Third, when the external constraints have the mandatory effect of specific information instructions, the system will leave the existing state and construct its own mode through otherorganizational methods according to the way and path provided by external constraints.

In this regard, external factors can provide external conditions for the development of system self-organization and provide choices for branch paths and directions of the orderly evolution for the development of system self-organization. In addition, the role of external factors can also destroy the original basis of self-organization development within the system, causing it to develop in the direction of disorganization, and even give the system a mandatory other-organization mode so as to change the existing basis and nature and open up a new evolutionary branch path and direction.

5.4. Good and Evil in Constraint and Freedom. In 2013, Luciano Floridi, a British philosopher, published "The Ethics of Information". In this book, he simply related the amount of system entropy to the ethical principles of good and evil and attempted to establish a universal macro-ethics on this basis. In relevant discussions, Mr. Floridi directly used the 


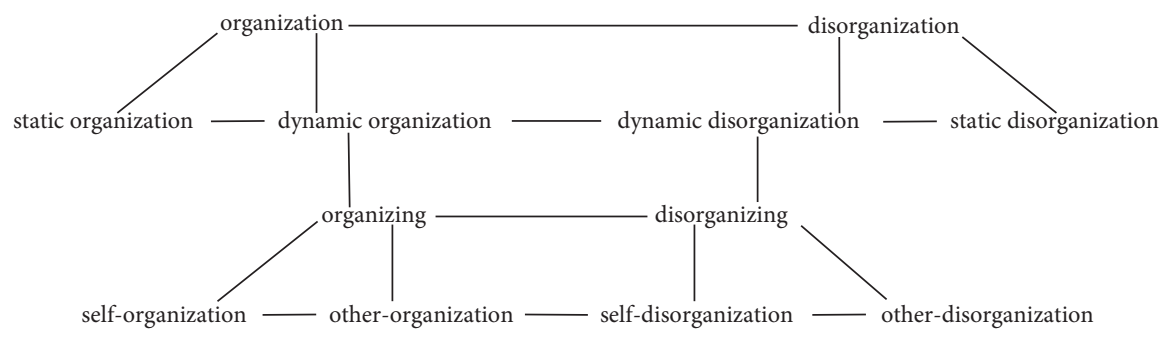

FIgURE 1: Conceptual systems of organization and disorganization.

quantitative measurement methods of entropy and information in information theory as the basic criterion of good or evil. The core view is to correspond "evil" with disorder, disordering and entropy increase, and "good" with order, ordering and entropy decrease. He clearly put forward a basic principle of the supreme good: eliminate existing entropy and prevent the generation of new entropy [15].

Based on the previous discussion of the relations and characteristics between organization and disorganization, order and disorder, entropy increase and entropy decrease, and constraint and freedom, it is easy to find the absurdity of Mr. Floridi's ethical principles. All the processes of organizing, ordering, and entropy decreasing increase the overall order, but at the same time they restrict, oppress, and enslave individuals. On the contrary, all processes of disorganizing, disordering, and entropy-increasing reduce the overall order but at the same time bring individuals a certain degree of freedom and liberation.

As far as the development of nature and society is concerned, excessive organizing and entropy decrease will lead to the exhaustion of development vitality, leading to rigidity and stagnation. On the contrary, excessive disorganizing and increasing entropy may bring chaos and turbulence that will also bring disastrous consequences to nature and society. "Entropy and entropy increase are not absolutely 'evil"'. Information and entropy decrease are not absolutely "good." A reasonable ethical principle should be compatible with seemingly opposing factors and maintain some reasonable tension between these opposing factors, for example, information and entropy, order and disorder, integrity and reductivity, certainty and nondeterminism, determinism and nondeterminism, purpose and randomness, necessity and contingency, and so on [16].

\section{Conceptual System Diagram of Organization and Disorganization}

Based on the above discussion, the many concepts related to organization and disorganization may be classified into four basic levels: Level 1: organization and disorganization; Level 2: static organization and dynamic organization and static disorganization and dynamic disorganization; Level 3: organizing and disorganizing can be distinguished under dynamic organization and disorganization; and Level 4: self-organization and other-organization and self-disorganization and other-disorganization can be distinguished under organizing and disorganizing.
Figure 1 represents this four-level structure. The connections indicate the complementarity, compatibility, and transformation between links.

\section{Data Availability}

The data used to support the findings of this study are available from the corresponding author upon request.

\section{Conflicts of Interest}

The authors declare that they have no conflicts of interest regarding the publication of this paper.

\section{References}

[1] G. Nicolis, Ilya Prigogine, Self-Organization in Non-equilibrium System, p. 60, John Wiley \& Sons, New York, 1977.

[2] H. Hermann, "Self-Organization" Synergetics an Introduction Nonequilibrium Phase Transitions And Self-Organization In Physics, Chemistry And Biology Published by, p. 191, SpringerVerlag Berlin Heidelberg, New York, 1978.

[3] D. Miao, "Self-organization and other-organization," Journal of Renmin University of China, vol. 4, pp. 67-70, 1988.

[4] T. Wu, "An introduction to self-organization methodology," Journal of Systematic Dialectics, vol. 2, pp. 4-10, 2001.

[5] X. Shen, T. Wu, and J. Yu, "From unorganized to organized, from organized to self-organized," Studies in Dialectics of Nature, vol. 8, pp. 122-126, 2013.

[6] W. Hofkirchner, "Does computing embrace self-organisation?," in Information and Computation, G. Dodig-Crnkovic and M. Burgin, Eds., World Scientific, Singapore, 2011.

[7] D. Miao, Research on Complexity Science, p. 136, China Books Publishing House, Beijing, China, 2014.

[8] N. Wiener, Cybernetics: Or Control and Communication in the Animal and the Machine, MIT Press, Cambridge, 1948.

[9] H. Hermann, "The Challenge of Complex Systems: Self-Organization" Information and Self-Organization A Macroscopic Approach to Complex Systems thep. 11, Third Edition, Springer-Verlag Berlin Heidelberg, NewYork, 2006.

[10] L. Gabora, "Self-other organization," Journal of Theoretical Biology, vol. 241, no. 3, pp. 443-450, 2006.

[11] T. Wu, "The problems of "I ching" changes, transfer changes and "existence" from the perspective of information philosophy," History of Chinese philosophy, vol. 73, no. 1, pp. 48-52, 2021.

[12] T. Wu, "New thinking on the problem of existence based on the theory of Information Philosophy -- the level of existence, existence and non-being," Studies on Dialectics of Nature, vol. 11, pp. 110-116, 2015. 
[13] E. Morin, LA MÉTHODE Tome 1 La Nature de la Nature, 2.L'organisation (de l'objet au système II.L'Unité complexe organisée. B.Les contraintes:le tout est moins que la somme des parties, Éditions du Seuil, Paris, France, 2008.

[14] N. Wiener, The human use of human beingsp. 51, Free Association, Great Britain, UK, 1989.

[15] L. Floridi, The Ethics of Information, pp. 71-101, Oxford University Press, Oxford, 2013.

[16] K. Wu and J. Wang, "Why is entropy not enough? - good emerges from the relaxation between order and disorder," Theoretical Information Studies, pp. 37-59, 2020. 